\title{
The extragalactic nature of GT $2318+620$
}

\author{
J. M. Paredes ${ }^{1}$, J. Martí ${ }^{2}$, W. K. Scott ${ }^{3}$, and R. K. Zamanov ${ }^{4}$ \\ 1 Departament d'Astronomia i Meteorologia, Universitat de Barcelona, Av. Diagonal 647, 08028 Barcelona, Spain \\ 2 Departamento de Física, Escuela Politécnica Superior, Universidad de Jaén, Virgen de la Cabeza 2, 23071 Jaén, Spain \\ e-mail: jmarti@ujaen.es \\ 3 Department of Physics and Astronomy, University of Calgary, 2500 University Dr. NW, Calgary, Alberta T2N 1N4, Canada \\ e-mail: bill@ras.ucalgary.ca \\ 4 Astrophysics Research Institute, Liverpool John Moores University, Twelve Quays House, Egerton Wharf, \\ Birkenhead CH41 1LD, UK \\ e-mail: rz@astro.livjm.ac.uk
}

Received 6 October 2003 / Accepted 1 March 2004

\begin{abstract}
We report optical and radio observations of GT 2318+620, a previously proposed variable radio source at low galactic latitude. With the information available more than a decade ago, this object was considered to be a low-mass X-ray binary with radio jets. However, the galactic nature of GT 2318+620 was never confirmed. The new data presented here provide substantial evidence to rule out a galactic origin and point towards identification as an active extragalactic source.
\end{abstract}

Key words. galaxies: active - X-rays: binaries

\section{Introduction}

GT $2318+620$ is an enigmatic source whose true nature has remained uncertain over the years. It was originally discovered by Gregory \& Taylor (1986) as a result of their galactic plane survey to search for variable radio sources. Several years later, Taylor et al. (1991) reported the detection of bipolar radio jets with the Very Large Array (VLA), extending over several arc-seconds, as well as neutral hydrogen absorption spectra suggesting a possible galactic origin. Based on this evidence, GT $2318+620$ was proposed to be a new X-ray binary system (of low mass) with relativistic jets, i.e., what today we know of as a microquasar. Unfortunately, the confirmation of the microquasar nature of GT $2318+620$ has remained elusive more than a decade and a half after the publication of the original papers. It is also intriguing that no detection in the $\mathrm{X}$-ray domain ever has been reported for this object. For instance, the source catalogs derived from the ROSAT All Sky Survey (Voges et al. 1999) reveal no X-ray emitter consistent with its position. Scott (1996) noted that the lack of any significant morphological changes, over several epochs at radio wavelengths, made GT 2318+620 more consistent with ordinary extragalactic sources, rather than a galactic X-ray binary. However, this object has remained poorly studied since.

In this paper, we present new observational evidence in the optical domain that clarifies the nature of GT $2318+620$ as an extragalactic source, and not a microquasar. In addition,

Send offprint requests to: J. M. Paredes,

e-mail: jmparedes@ub.edu some previously unpublished radio observations of this source are provided.

\section{Observations and results}

\subsection{Radio observations}

We report here on some radio images of GT $2318+620$ obtained by us several years ago with VLA interferometer of the National Radio Astronomy Observatory (NRAO) in the USA. The VLA observations were conducted in two different epochs using the most compact and the most extended configuration of the array, D and A respectively. All VLA data were edited, calibrated and imaged using the NRAO AIPS software package.

The radio field around GT $2318+620$ is displayed in Fig. 1 as observed on January 11, 1994 at the $3.5 \mathrm{~cm}$ wavelength in the $\mathrm{D}$ configuration. The target is very close to a neighbouring double radio source, already noted by Taylor et al. (1991) about $3^{\prime}$ South West. The coordinate system for all our map axes is B1950.0, which was the standard used when the data were obtained. The contour plot in Fig. 1, corresponding to the compact D configuration, has a good sensitivity to extended emission and reveals that the GT $2318+620$ jets extend over nearly one arc-minute. Possible hints of precession are also detected especially in the southern jet, which appears to be curving. The total flux density of the source is $S_{3.5} \mathrm{~cm}=8.2 \pm 0.1 \mathrm{mJy}$ and most of it is located at the central core.

Figure 2 displays a VLA map obtained in July 1991 using the A configuration of the array. The corresponding contour 


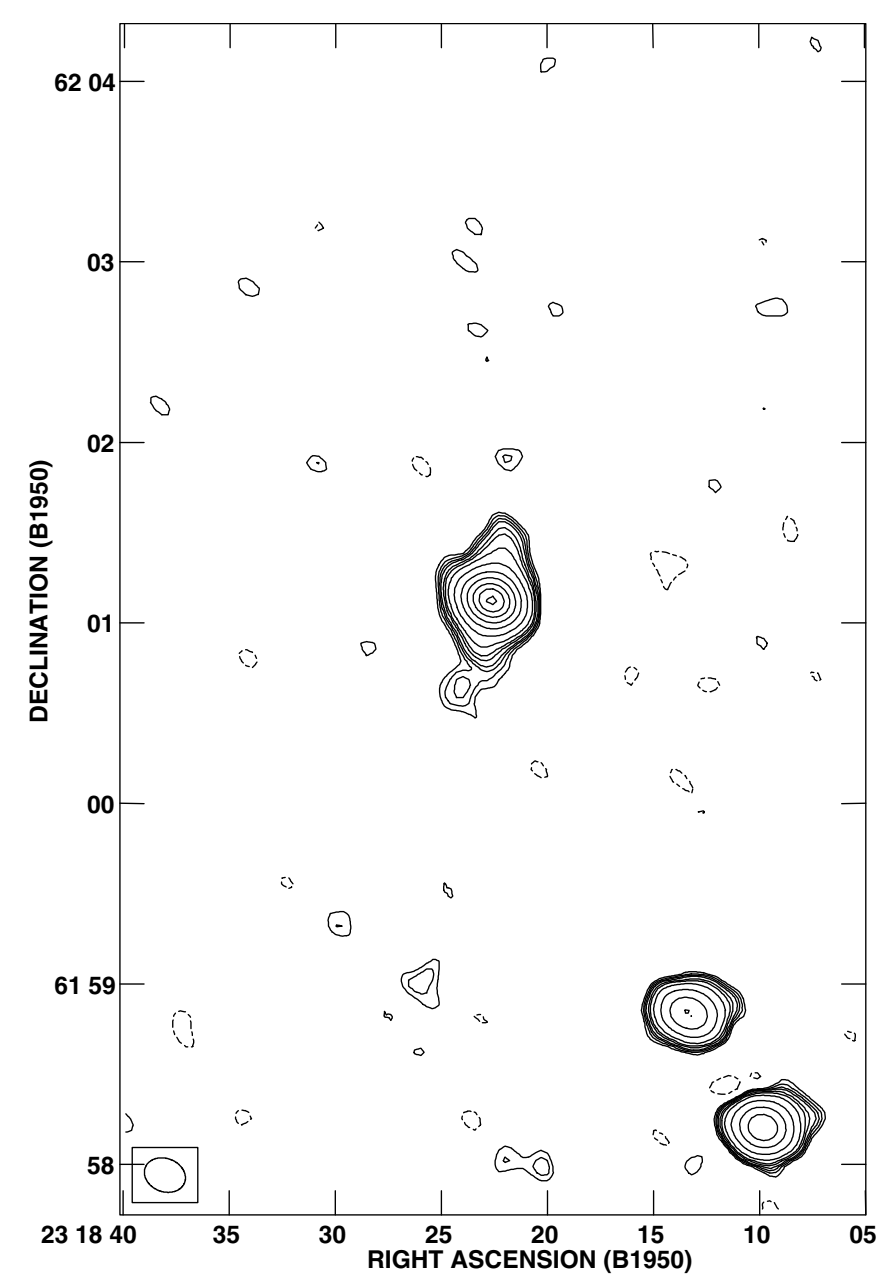

Fig. 1. Radio map of the field around GT $2318+620$ obtained with the VLA on January 11, 1994 at the $3.5 \mathrm{~cm}$ wavelength in D configuration. The contours shown are $-3,4,5,6,8,10,15,30,60,100,150$, 200, 250 and 300 times the r.m.s. noise of $0.025 \mathrm{mJy}_{\text {beam }}{ }^{-1}$. The corresponding synthesised beam is $14 .^{\prime \prime} 0 \times 11$." $^{\prime} 1$ at a position angle of $68^{\circ} .6$.

plot reveals the GT 2318+620 morphology with arc-second angular resolution. The central core of the source and its bipolar jet are clearly evident. The total flux density of the source in this image is $S_{20 \mathrm{~cm}}=16 \pm 2 \mathrm{mJy}$. The position of the core, with peak flux density of $6.3 \pm 0.1 \mathrm{mJy} \mathrm{beam}^{-1}$, is found to be consistent with that reported by Taylor et al. (1991). The original position given by these authors was also in the B1950.0 system and we have precessed it to J2000.0, with the result being $\alpha_{\mathrm{J} 2000.0}=23^{\mathrm{h}} 20^{\mathrm{m}} 34^{\mathrm{s}} .109 \pm 0.02$ and $\delta_{\mathrm{J} 2000.0}=$ $+62^{\circ} 17^{\prime} 33$." $45 \pm 0$ ' $^{\prime \prime} 15$. Hereafter, this is the radio position that we will use for astrometric purposes at optical wavelengths.

\subsection{Radio monitorings}

GT 2318+620 was monitored daily with the Green Bank Interferometer during 1996-1998 at 2.25 and $8.3 \mathrm{GHz}$ (11 and $3.6 \mathrm{~cm}$ ). Inspection of this public data reveals a persistent emission level over the years. Typical flux densities during this period were $S_{11 \mathrm{~cm}} \simeq 40 \mathrm{mJy}$ and $S_{3.6 \mathrm{~cm}} \simeq 10 \mathrm{mJy}$ at 2.25 and $8.3 \mathrm{GHz}$, respectively. Possible variability could be present in

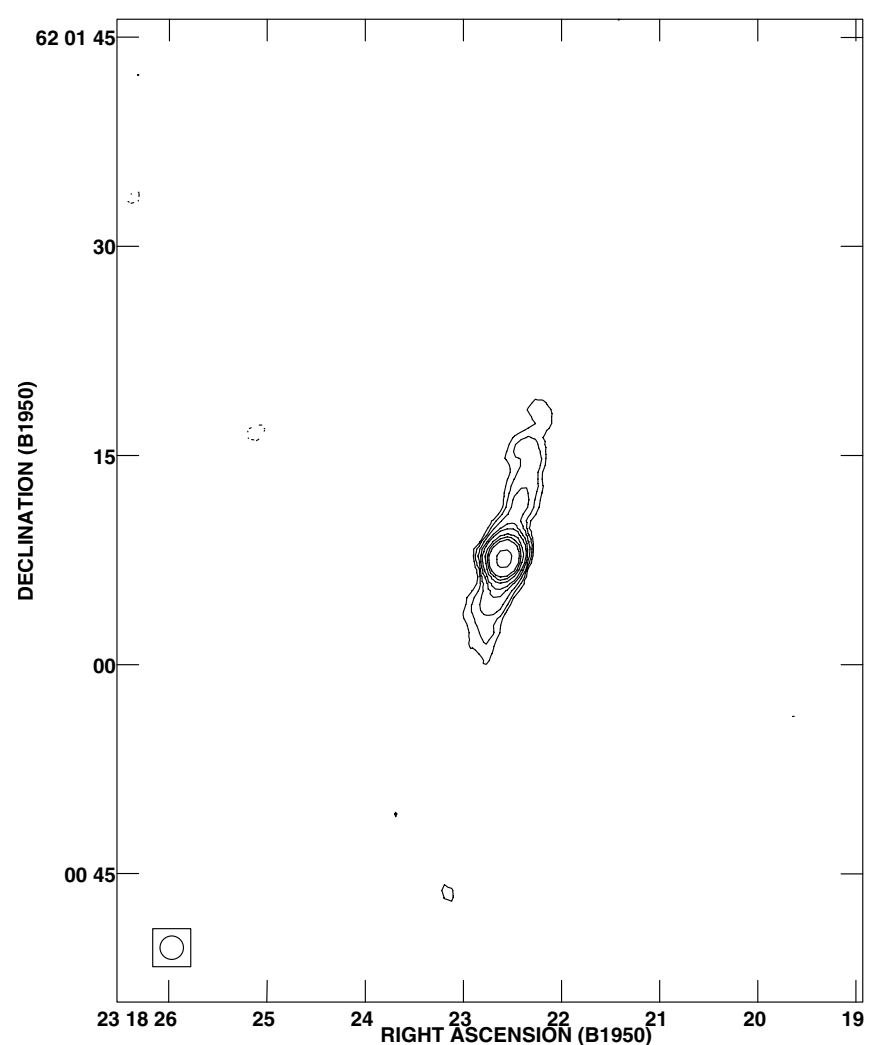

Fig. 2. Naturally weighted map of GT $2318+620$ obtained in A configuration on July 291991 at $20 \mathrm{~cm}$. It clearly shows both the central core and the bipolar jets with arc-second angular resolution. The corresponding synthesised beam is $1 . " 7 \times 1$." 7 , practically circular. The contours shown are $-0.25,0.25,0.35,0.5,0.75,1,1.5,2,2.5$ and $5 \mathrm{mJy}^{\text {beam }^{-1}}$.

the GBI data, but most of it is likely induced by confusion effects due to the nearby double source (see Fig. 1) inside the GBI primary beam (measuring 22' at $2.25 \mathrm{GHz}$ and 5.8 at $8.3 \mathrm{GHz}$ ). On the other hand, there is no evidence of strong flaring events, being the flux density jumps in the GBI light curve related to changes of the observation hour angle of the interferometer. In view of this facts, the GBI radio light curves cannot be considered very reliable for this source and will not be considered further.

Similar confusion or hour angle effects, or small pointing errors as well, may have caused the classification of GT $2318+620$ as a possible short term variable by Gregory $\&$ Taylor (1986) in their original survey carried out with the NRAO $91 \mathrm{~m}$ transit telescope, which had two 2'.8 beams separated by $7 ! 2$. These authors defined two short term variability indices $(V 1$ and $V 2)$ which were sensitive to continuous and episodic variability, respectively. GT $2318+620$ was reported to have $V 1=1.76$ and $V 2=2.35$ while a true variable was considered to have $V 1 \geq 1.85$ and/or $V 2 \geq 3.5$, corresponding to a probability of obtaining by chance the measured $V 1$ or $V 2$ values smaller than $0.1 \%$.

GT 2318+620 was also ocasionally monitored with the $70 \mathrm{~m}$ antenna at the Madrid Deep Space Communication Complex (MDSCC) of INTA-NASA in Spain, using the $3.6 \mathrm{~cm}$ receivers. The beam size was 2 '. 1 thus safely excluding the 
Table 1. Flux densities of GT $2318+620$.

\begin{tabular}{lccc}
\hline \hline Observation & $\begin{array}{c}\lambda \\
(\mathrm{cm})\end{array}$ & $\begin{array}{c}\text { Flux density } \\
(\mathrm{mJy})\end{array}$ & Remarks \\
\hline Taylor et al. (1991) & 21 & $15 \pm 2$ & WSRT, total \\
& 20 & $37 \pm 2$ & VLA, total \\
& 20 & $6.3 \pm 0.3$ & VLA, core \\
& 6 & $7.7 \pm 0.3$ & VLA, core \\
\hline Condon et al. (1998) & 20 & $14.2 \pm 0.6$ & NVSS, total \\
\hline This paper & 20 & $16 \pm 2$ & VLA, total \\
& 20 & $6.3 \pm 0.1$ & VLA, core \\
& 3.5 & $8.2 \pm 0.1$ & VLA, total \\
& 3.6 & $9.0 \pm 2.5$ & MDSCC, total \\
& 3.6 & $8.3 \pm 2.5$ & ", \\
\hline
\end{tabular}

nearby confusing double. Both right and left circular polarization were observed at the same time using a dual band reflex feed system (Rusch 1976). The details of the observation procedure can be found in Paredes et al. (1990). The data were reduced using the software package available at the telescope site (Rius et al. 1988). The source was detected at an average flux density level of $S_{3.6 \mathrm{~cm}} \simeq 9.0 \pm 2.5 \mathrm{mJy}$ in February 1993 and $S_{3.6 \mathrm{~cm}} \simeq 8.3 \pm 2.5 \mathrm{mJy}$ in March 1995 . These values agree with those measured by the VLA and GBI at almost the same wavelength.

In Table 1 we have collected all available radio flux densities of GT 2318+620 excluding the GBI data for the reasons quoted above. It is interesting to point out that all total flux density values obtained at $20 \mathrm{~cm}$ with different interferometers over the years (WSRT, NVSS and our VLA data) agree around $S_{20 \mathrm{~cm}} \simeq 15 \mathrm{mJy}$. The only exception is the $37 \mathrm{mJy}$ point from the Taylor et al. (1991) paper. However, this measurement may not be accurate due to possible cleaning artifacts in their Fig. 2a. Therefore, we suggest that GT 2318+620 is more likely to be a constant radio source than a short term variable. The agreement between the $20 \mathrm{~cm}$ core flux densities in Table 1 and those at 3.5/3.6 cm from our VLA and MDSCC data also supports the non-variable nature of this source.

If this is correct, we can compute the radio spectral index $\left(S_{v} \propto v^{\alpha}\right)$ using the non-simultaneous flux densities in Table 1. From the most accurate NVSS $20 \mathrm{~cm}$ and VLA $3.5 \mathrm{~cm}$ values, the overall spectral index is estimated to be $\alpha=-0.32 \pm 0.03$. Similarly, the most accurate $20 \mathrm{~cm}$ and $6 \mathrm{~cm}$ values indicate a core spectral index slightly inverted with $\alpha=+0.17 \pm 0.03$.

\subsection{Optical observations}

In 2002, we carried out a series of both imaging and spectroscopic observations of GT $2318+620$ at optical wavelengths. We used the $4.2 \mathrm{~m}$ William Herschel Telescope (WHT) at the Roque de los Muchachos observatory in Canary Islands (Spain) in visitor mode. The ISIS double-armed spectrograph was mounted at the Cassegrain focus of the telescope with different gratings in the red and blue arms, for a spectral resolution of 5 to $10 \AA$. The images were obtained using the AUX camera simultaneously available with ISIS. The technical details of the WHT instrumentation are described in the ISIS manual (Carter et al. 1994). All frames were processed using the IRAF

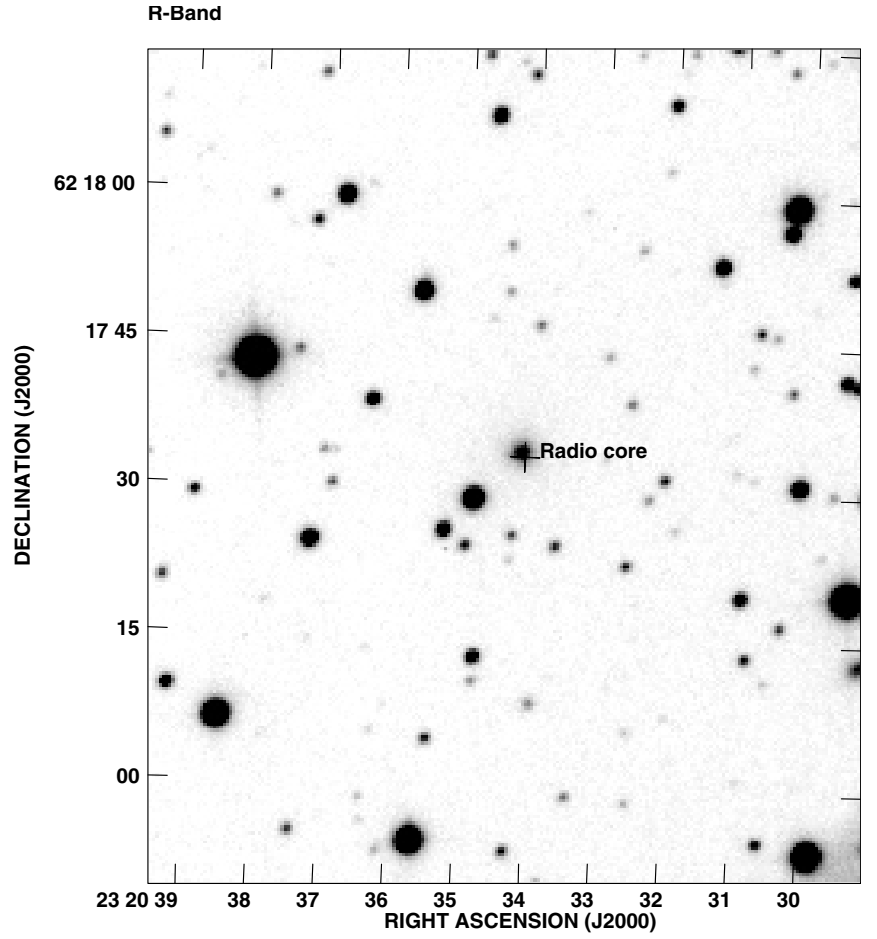

Fig. 3. Optical $R$-band image of the field around GT $2318+620$ obtained in 2002 December 4 with the WHT and $300 \mathrm{~s}$ integration time. The image seeing is $0{ }^{\prime \prime} 90 \pm 0 . \prime 01$ ( $F W H M$ of stellar profiles). The small cross indicates the position of the radio core according to our astrometric solution.

package, including bias subtraction and flat field correction. The spectra were further extracted and flux calibrated using spectroscopic standard stars.

In Fig. 3, we present a $R$-band CCD image of GT $2318+620$ taken with sub-arcsecond seeing. The central cross indicates the position of the radio core as quoted previously. The astrometric grid in the WHT image was derived from a set of 6 USNO A2.0 stars (Monet et al. 1999) in the CCD field. The residuals of the astrometric fit do not exceed $\sim 0$.' 2 , while the error for the USNO A2.0 positions is about 0.' 25 . The ISIS red arm spectrum of the GT 2318+620 optical counterpart is shown in Fig. 4, taken with the R316R grating at a dispersion of $62 \AA \mathrm{mm}^{-1}$.

\section{Discussion}

The radio results reported here represent a substantial change in our understanding of GT $2318+620$. The original reason for all the interest in this source, radio variability, is no longer confirmed. This fact casts some doubts about GT $2318+620$ being a microquasar in the Galaxy because galactic jet sources are often variable at radio wavelengths at least with moderate amplitude. The possibility of GT $2318+620$ being an extragalactic object appears then as a very reasonable alternative and the optical observations discussed below actually support this interpretation.

In the WHT image of Fig. 3 there is clearly an optical source close to the radio position. Its approximate magnitude is estimated to be $R=18.9 \pm 0.1$, using the photometric zero 


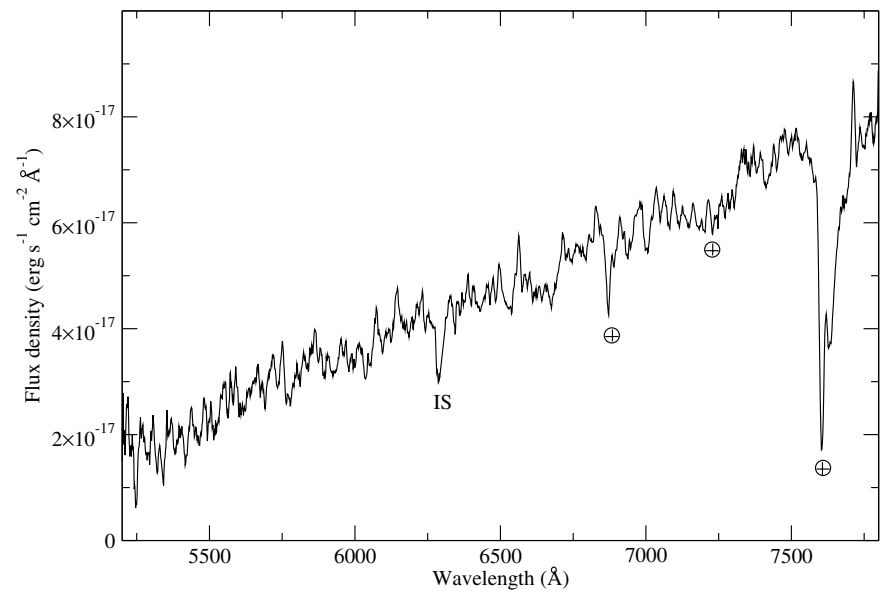

Fig. 4. Optical spectrum of GT $2318+620$ resulting from the average of two spectra taken on December 3 and 4, 2002. Each individual spectrum was obtained with the R316R grating, each of them with an integration time of $2000 \mathrm{~s}$. The only significant features detected are of interstellar and telluric origin. The ISIS blue arm data is not shown because of very low signal-to-noise ratio.

point derived from the only standard from Landolt et al. (1992) that we could observe. From our CCD astrometry, the optical position is measured to be $\alpha_{\mathrm{J} 2000.0}=23^{\mathrm{h}} 20^{\mathrm{m}} 34^{\mathrm{s}} .16 \pm 0.04$ and $\delta_{\mathrm{J} 2000.0}=+62^{\circ} 17^{\prime} 34^{\prime \prime}$. $0 \pm 0 .^{\prime \prime} 3$. The offsets between the optical and radio position are thus $\Delta \alpha \cos \delta=0$ ". 36 and $\Delta \delta=0$.' 55 . Therefore, their coincidence is acceptable within astrometric error.

We thus confirm the existence of an optical counterpart to GT $2318+620$ as proposed by Taylor et al. (1991), based on their inspection of Palomar plates. The counterpart was also found to be present in archive CCD I-band frames taken in August 14/15 1994 at the $2 \mathrm{~m}$ telescope of the Bulgarian National Astronomical Observatory "Rozhen" with exposure of $1200 \mathrm{~s}$. The estimated magnitude was $I=17.6 \pm 0.2$, based on aperture photometry and standards in M 67 (Chevalier \& Ilovaisky 1991) and NGC 7709 (Christian et al. 1985).

Moreover, the optical counterpart has an interesting extended appearance. This can be clearly seen in the contour plot of Fig. 5, which is an enlargement of the same $R$-band CCD frame taken with the WHT. An elliptical Gaussian fit gives an apparent angular size of $\left(1^{\prime \prime} 58 \pm 0.04\right) \times\left(1{ }^{\prime \prime} 48 \pm\right.$ $\left.0 .^{\prime \prime} 04\right)$, with position angle of $106^{\circ} \pm 16^{\circ}$. These dimensions are clearly above the seeing disk of $0 .^{\prime \prime} 90 \pm 00^{\prime \prime} 01$ (Full Width Half Maximum, $F W H M$ ) estimated from similar Gaussian fits on several stellar point sources in the field.

The measurement of an apparent angular size for the GT $2318+620$ optical counterpart strongly rules out a galactic stellar object. On the contrary, the most natural interpretation is that we are dealing with an extragalactic source that is resolved under good seeing conditions.

In Fig. 6, we have plotted the surface brightness profile of GT $2318+620$ as a function of the angular distance $r$ in arcseconds against a de Vaucouleurs law, $I(r)=I(0) \mathrm{e}^{-k r^{1 / 4}}$, typical of elliptical galaxies, convolved with the seeing disc (assumed to be a circular Gaussian with FWHM 0.'9). The similarity

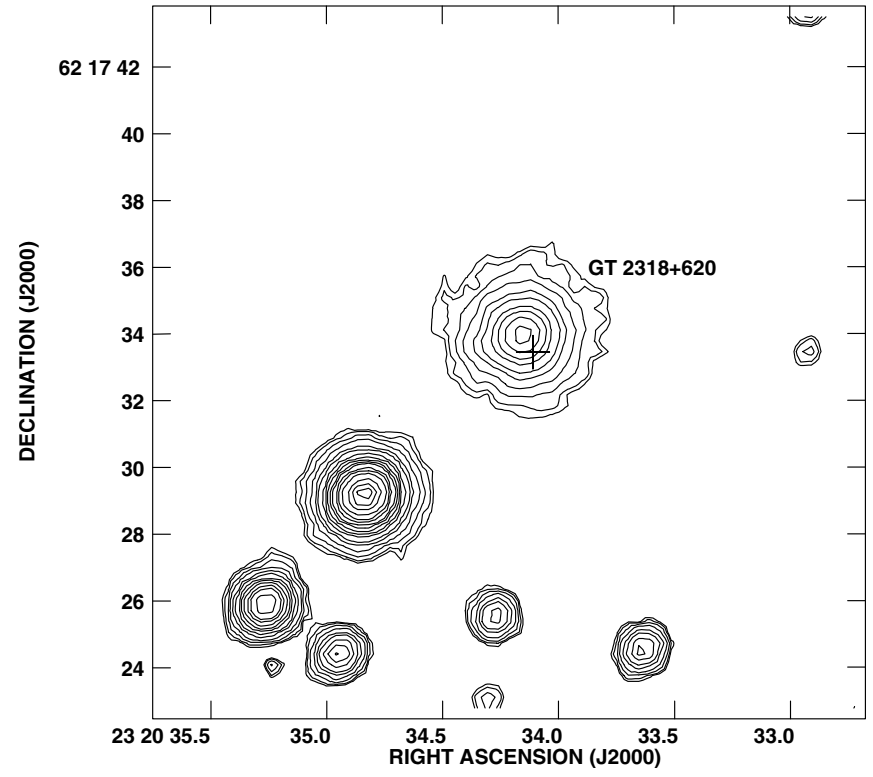

Fig. 5. Contour plot showing an enlarged view of Fig. 3. The extended appearance of GT $2318+620$ is clearly evident when compared with the nearby stars. The small cross indicates the position of the VLA radio core.

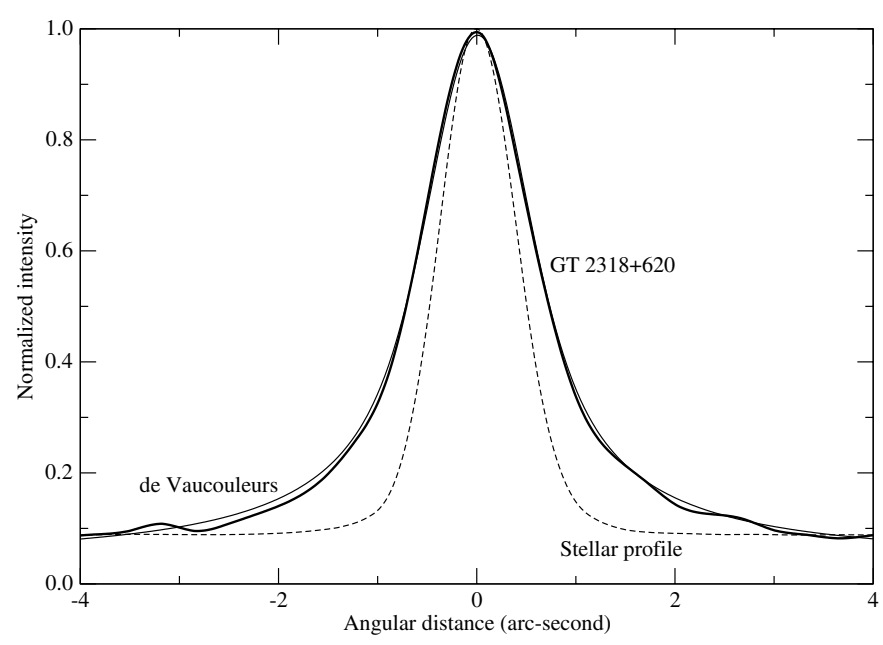

Fig. 6. Surface brightness profile in the East-West direction of GT 2318+620 (thick line) compared with a stellar profile (dashed line) and a de Vaucouleurs law convolved with a Gaussian seeing disk with FWHM of 0.'9 (solid line).

between the observed and the convolved profiles strongly supports that GT $2318+620$ is in fact an elliptical galaxy.

The optical spectrum in Fig. 4 shows no hint of stellar photospheric features, which is consistent with an extragalactic object. It displays an almost featureless continuum with a clear and broad absorption feature appearing at about $6280 \AA$ (equivalent width $\simeq 10 \AA$ ). A tentative interpretation is that this corresponds to the superposition of the $6284 \AA$ interstellar band and redshifted Na I $5893 \AA$ doublet from stars and interstellar gas in GT $2318+620$. If this is correct, the redshift is crudely estimated to be $z=0.066$ corresponding to a luminosity distance of about $290 \mathrm{Mpc}$ (assuming the Hubble constant to be $H_{0}=71 \mathrm{~km} \mathrm{~s}^{-1} \mathrm{Mpc}^{-1}$ based on WMAP precision 
cosmology). From values in Table 1, the corresponding radio luminosity at this distance would be then $L_{21} \mathrm{~cm} \simeq 1.5 \times$ $10^{23} \mathrm{~W} \mathrm{~Hz}^{-1}$. This would suggest a low power Fanaroff Riley I (FR I) galaxy considering the radio morphology of the jet source brighther to the center (Fanaroff \& Riley 1974). FRIs do not usually show emission lines in their spectra, in agreement with our observations. The linear size of the Galaxy is estimated to be $\sim 100 \mathrm{kpc}$, or larger, depending on jet inclination.

The strong absorption towards the blue in our WHT spectrum could be in part attributed to an extragalactic object seen through the Galactic Plane. The HI survey by Dickey \& Lockman (1990) gives a column density of $N_{\mathrm{H}} \simeq 8.7 \times$ $10^{21} \mathrm{~cm}^{-2}$ at the GT $2318+620$ position. According to Predelh \& Schmitt (1995), this is equivalent to an optical extinction $A_{\mathrm{V}}=0.56\left(N_{\mathrm{H}} / 10^{21} \mathrm{~cm}^{2}\right)+0.23=5.1 \mathrm{mag}$, or higher since only the hydrogen atomic component is considered. However, this survey has a very low angular resolution $\left(\sim 1^{\circ}\right)$ and could be not strictly reliable at a particular position in the sky. Taylor et al. (1991) report a column density of $1.5 \times 10^{21} \mathrm{~cm}^{-2}$ at the precise location of GT $2318+620$, which translates into a much lower extinction value of $A_{\mathrm{V}}=1.1 \mathrm{mag}$. Finally, we performed the exercise of fitting an elliptical galaxy spectral template from Storchi-Bergmann et al. (1998) to our observed spectrum in Fig. 4. Several values of $A_{\mathrm{V}}$ from 1 to $10 \mathrm{mag}$ were tried and the slope of the continuum was actually better reproduced around $A_{\mathrm{V}} \simeq 5 \mathrm{mag}$. Therefore, the true extinction value towards GT $2318+620$ is likely in the range 1 to $5 \mathrm{mag}$, with $A_{\mathrm{V}} \simeq 2-3$ mag being a reasonable guess.

Additional observations will be needed to better constrain the distance and extinction properties of GT 2318+620. Independently of this however, its extragalactic origin and elliptical galaxy classification are well established now.

\section{Conclusions}

We have presented new observations of GT $2318+620$ which argue against this object being a galactic $\mathrm{X}$-ray binary with relativistic jets and a variable radio source. On the contrary GT $2318+620$ is more likely to be an extragalactic object, possibly an elliptical FR I radio galaxy. The key fact supporting the proposed extragalactic nature is the extended appearance of the optical counterpart, whose surface brightness profile is well represented by a de Vaucouleurs law convolved with the seeing disc. The spectroscopic observations indicate an almost featureless and absorbed spectrum $\left(A_{\mathrm{V}} \sim 2-3 \mathrm{mag}\right)$ with a possible redshift of $z=0.066$.
Acknowledgements. We thank our referee Dr. Richard Hunstead for his useful comments that helped to improve this paper. The National Radio Astronomy Observatory is a facility of the National Science Foundation operated under cooperative agreement by Associated Universities, Inc. in the USA The William Herschel Telescope is operated on the island of La Palma by the Isaac Newton Group in the Spanish Observatorio del Roque de los Muchachos of the Instituto de Astrofísica de Canarias. J.M.P. and J.M. acknowledge partial support by DGI of the Ministerio de Ciencia y Tecnología (Spain) under grant AYA2001-3092, as well as partial support by the European Regional Development Fund (ERDF/FEDER). J.M. has been aided in this work by an Henri Chrétien International Research Grant administered by the American Astronomical Society. J.M. is also supported by the Junta de Andalucía (Spain) under project FQM322. The Green Bank Interferometer is a facility of the National Science Foundation operated by the NRAO in support of NASA High Energy Astrophysics programs. We are finally grateful to the staff of the NASA-DSN Madrid Deep Space Communication Complex for their support in carrying out monitoring radio observations.

\section{References}

Carter, D., Benn, C. R., Rutten, R. G. M., et al. 1994, ISIS DOUBLE BEAM SPECTROGRAPH, version 1.0, http://www.ing.iac.es/Astronomy/instruments/isis

Chevalier, C., \& Ilovaisky, S. A. 1991, A\&AS, 90, 225

Christian, C. A., Adams, M., Barnes, J. V., et al. 1985, PASP, 97, 363

Condon, J. J., Cotton, W. D., Greisen, E. W., et al. 1998, AJ, 115, 1693

Dickey, J. M., \& Lockman, F. J. 1990, ARA\&A, 28, 215

Fanaroff, B. L., \& Riley, J. M. 1974, MNRAS, 167, 31

Gregory, P. C., \& Taylor, A. R. 1986, AJ, 92, 371

Landolt, A. U. 1992, AJ, 104, 340

Monet, D. G., Bird, A., Canzian, B., et al. 1999, USNO-A2.0 CD-ROM (Washington DC: US Naval Observatory)

Paredes, J. M., Estalella, R., \& Rius, A. 1990, A\&A, 232, 377

Predehl, P., \& Schmitt, J. H. M. M. 1995, A\&A, 293, 889

Rius, A., Chamarro, A., Estalella, R., et al. 1988, Data Gathering System for Radio Astronomical Applications, MDSCC, Madrid

Rusch, W. V. T. 1976, in Methods of Experimental Physics 12, Part B, ed. M. L. Meeks (New York: Academic Press), 29

Scott, W. K. 1996, Ph.D. Thesis, University of British Columbia

Storchi-Bergmann, T., Calzetti, D., \& Kinney, A. L. 1998, A Database of UV-Optical Spectra of Nearby Quiescent and Active Galaxies, http://www. stsci. edu/ftp/catalogs/nearby_gal/sed.html

Taylor, A. R., Gregory, P. C., Duric, N., \& Tsutsumi, T. 1991, Nature, 351,547

Voges, W., Aschenbach, B., Boller, Th., et al. 1999, A\&A, 349, 389 